VOLUMEN 37 | N 1 | ABRIL 2019

FECHA DE RECEPCIÓN: : 08/01/2019 FECHA DE APROBACIÓN: 24/04/2019 FECHA PUBLICACIÓN: 30/04/2019

1. MSP Hospital Vicente Corral Moscoso

2. MSP Hospital Isidro Ayora de Loja

3. Universidad de Cuenca

\begin{tabular}{l|l} 
Caso & Clinical \\
Clínico & Case
\end{tabular}

Correspondencia:

md.franciscevallosdagmail.com

Dirección:

Av. Loja 5-42 y Alonso Quijano

Código Postal:

010201

Teléfonos:

(07)24034863 - 0984140564

Cuenca - Ecuador

\section{Insuficiencia cardíaca aguda tras administración de propofol y fentanilo en pacientes sin enfermedad cardíaca estructural: reporte de dos casos.}

\author{
Acute heart failure following propofol and fentanyl adminis- \\ tration in patients without structural heart disease: two cases \\ report. \\ Cevallos Sacoto Francisco Antonio', Puga Bravo Cristina Evelyn², \\ Serrano Béjar Leonardo Alfredo ${ }^{3}$, Tirapé Castro Hugo Arturo'
}

\section{RESUMEN}

La insuficiencia cardíaca aguda se presenta excepcionalmente en pacientes sin enfermedad cardíaca estructural. Se presenta dos casos clínicos con insuficiencia cardiaca luego de intervención quirúrgica en donde se administraron dosis de propofol y fentanilo, fármacos relacionados con efecto depresor cardiovascular; se observa un deterioro hemodinámico súbito, con disminución de la fracción de eyección que en el seguimiento regresaría a parámetros de normalidad. Al descartarse patología orgánica de corazón, el uso de estos fármacos se relaciona con el desarrollo de la insuficiencia cardiaca, por lo que se propone tenerlo en cuenta en el diagnóstico diferencial de esta patología.

Palabras clave: informes de casos, insuficiencia cardíaca, fentanilo, propofol, combinación de medicamentos.

\section{ABSTRACT}

Acute heart failure occurs exceptionally in patients without structural heart disease. Two clinical cases with heart failure after surgical intervention were presented, in which doses of propofol and fentanyl were administered, drugs related to cardiovascular depressant effect; a sudden hemodynamic deterioration is observed, with a decrease in the ejection fraction, which in the monitoring would return to normal parameters. When discarding organic pathology of heart, the use of these drugs is related to the development of heart failure, so it is proposed to take it into account in the differential diagnosis of this condition.

Keywords: case reports, heart failure, fentanyl, propofol, drug combinations. 


\section{INTRODUCCIÓN}

La insuficiencia cardíaca aguda (ICA) se define como la aparición rápida o el empeoramiento de los síntomas o signos de insuficiencia cardíaca. Es una entidad potencialmente mortal que requiere evaluación y tratamiento urgentes. El edema agudo de pulmón y el choque cardiogénico son dos de sus principales presentaciones, siendo el tratamiento de esta ultima un verdadero reto para el personal médico [1].

Es conocido que el síndrome coronario agudo constituye la principal causa de ICA, sin embargo existen otras etiologías como: taquiarritmias, aumento excesivo de la presión arterial, infecciones, bradiarritmias, fármacos, así como la cirugía y sus complicaciones perioperatorias [1].

El uso de fármacos utilizados para sedación y analgesia, como el propofol y el fentanilo que deprimen la función miocárdica, repercutirían en el performance cardíaco, que no pasa de ser leve en pacientes con función y estructura cardiaca normal. El desarrollo de cuadros severos de ICA en pacientes con estas condiciones es muy infrecuente [2].

\section{Presentación de Casos}

\section{Primer caso}

Paciente femenina de 45 años de edad, de etnia mestiza, quien presentó edema agudo de pulmón de manera súbita después de una intervención quirúrgica de riesgo intermedio. Su historia médica reflejó antecedente de epilepsia en tratamiento con fenobarbital. Con ASAPS (por sus siglas en inglés "American Society of Anesthesiologists (ASA) physical status classificationsystem") de 2 y un peso de $75 \mathrm{~kg}$. La cirugía realizada fue colecistectomía laparoscópica por diagnóstico de pólipo vesicular. Se realizó pre medicación con: midazolam $2.5 \mathrm{mg}$. Inducción anestésica con: propofol $150 \mathrm{mg}$, fentanilo $300 \mu \mathrm{g}$, lidocaína $100 \mathrm{mg}$, atracurio $25 \mathrm{mg}$. Durante la cirugía la paciente se mantuvo hemodinámicamente estable. El proceder quirúrgico y anestésico concluyó sin complicaciones, el tiempo quirúrgico fue de 40 minutos. Se administró profilaxis antiemética con ondansetrón $4 \mathrm{mg}$, se extubó $\mathrm{y}$ traslado a la unidad de cuidados posoperatorios donde presentó súbitamente cuadro compatible con edema agudo de pulmón normotensivo que respondió adecuadamente al tratamiento con ven- tilación no invasiva (presión positiva continua en la vía aérea, CPAP por sus siglas en inglés), diuréti$\cos$ (furosemida) y vasodilatadores (nitroglicerina). Con acidosis metabólica en la gasometría arterial. El electrocardiograma taquicardia sinusal, intervalo P-R e intervalo QT normales, no alteraciones isquémicas y un trastorno de la conducción de rama derecha de nueva aparición. Signos de insuficiencia cardiaca fueron encontrados en la radiografía de tórax. Un ecocardiograma transtorácico (ETT) de urgencia reveló hipocinesia en los segmentos septales a nivel basal y medio, con fracción de eyección del ventrículo izquierdo (FEVI) limítrofe. Los resultados de hemograma, química básica y coagulograma resultaron normales. Posterior se realizó prueba de sensibilidad exponiendo por vía cutánea anestésicos utilizados, resultando negativa. La evolución fue favorable y posterior a realizarse un ETT que resulto normal se decidió alta hospitalaria.

\section{Segundo caso}

Paciente femenina de 41 años de edad, de etnia mestiza, quien presento ICA manifestada como choque cardiogénico de manera súbita después de una intervención quirúrgica de riesgo intermedio. Con historia médica de hipertiroidismo en tratamiento con propiltiouracilo, propanolol. Con ASAPS de 2 y un peso de $80 \mathrm{Kg}$. Se realizó colecistectomía laparoscópica por diagnóstico de colecistopatía alitiásica. Inducción anestésica con: propofol $200 \mathrm{mg}$, lidocaína $60 \mathrm{mg}$, fentanilo $300 \mu \mathrm{g}$ y atracurio $50 \mathrm{mg}$. Luego de realizar el neumoperitoneo la paciente presenta bradicardia sinusal a una frecuencia de 34 latidos por minuto (Ipm) tras la administración de $1 \mathrm{mg}$ de atropina, la frecuencia cardíaca se incrementa a $165 \mathrm{lpm}$ y se observan múltiples contracciones supraventriculares prematuras, este patrón resuelve con 80 mg de lidocaína, observándose un ritmo sinusal a una frecuencia de $90 \mathrm{lpm}$. Durante la cirugía se mantiene hemodinámicamente estable. El tiempo quirúrgico fue de 30 minutos. Se extuba sin complicaciones. En la sala de recuperación, presenta nausea, vértigo, disnea intensa, se auscultan estertores crepitantes bilaterales y tensión arterial sistólica de $80 \mathrm{mmHg}$, es decir un cuadro franco de edema agudo de pulmón hipotensivo, se inicia tratamiento con oxígeno por CPAP, apoyo inotrópico y diuréticos (furosemida) a dosis mínimas priorizando una tensión arterial media mayor a $60 \mathrm{mmHg}$. El cuadro progresa hasta establecerse un choque cardiogénico por lo que se 
inicia ventilación mecánica invasiva y se optimiza el apoyo inotrópico con infusión de dobutamina y dopamina, además en dependencia de las cifras de presión arterial se utilizó nitroglicerina y morfina.

En la medición de gases arteriales se observa acidosis metabólica compensada con hipoxemia leve. El electrocardiograma taquicardia sinusal con intervalos PR y QT normales, sin alteraciones isquémicas. En la radiografía de tórax se evidencian signos de insuficiencia cardiaca. El ecocardiograma transtorácico de urgencia reportó una disfunción sistólica severa (FEVI del 30\%), disfunción diastólica grado II con presión de la aurícula izquierda elevada. Trastornos importantes de la contractibilidad segmentaria, con acinesia de los segmentos lateral, medio y septal a nivel basal y medio. La evolución fue favorable, dentro de las siguientes 24 horas se retira paulatinamente el apoyo inotrópico y se repite ecocardiograma transtorácico donde se observa una FEVI del $55 \%$, y contractibilidad global y segmentaria adecuada al reposo, además de cavidades cardiacas de tamaño normal. Por lo que es dada de alta.

\section{DISCUSIÓN}

La insuficiencia cardiaca aguda se puede presentar como primera manifestación de la insuficiencia cardíaca (de novo) o ser la consecuencia de una descompensación aguda de un trastorno crónico; y, puede estar causada por una disfunción cardiaca primaria o precipitada por factores extrínsecos siendo el más frecuente la IC crónica [1]. En los casos presentados, se descarta el antecedente de insuficiencia cardiaca crónica por la clínica y por mostrar un ecocardiograma transtorácico (ETT) normal al alta hospitalaria.

La miocarditis viral pudiese estar relacionado con estos dos casos, sin embargo la ausencia de historia de infección reciente, hace a este diagnóstico improbable, aunque al no disponer de serología para virus relacionados con esta entidad, así como de resonancia magnética nuclear, no podríamos descartarla completamente [3]. Se puede sospechar un cuadro de miocardiopatía periparto que puede presentarse hasta en los cinco meses posteriores al parto y que cursa con cuadros súbitos de ICA y disfunción sistólica severa generalmente transitoria, se descarta esta posibilidad por la ausencia de embarazo reciente [4].
El síndrome de Takotsubo es un síndrome cardiaco agudo que simula un síndrome coronario agudo con elevación del segmento ST, caracterizado por anormalidades transitorias de la contractibilidad segmentaria que involucran los segmentos apicales y medios del ventrículo izquierdo en ausencia de enfermedad coronaria obstructiva significativa. Se produce predominantemente en mujeres posmenopáusicas, pero también puede ocurrir en mujeres más jóvenes. En el $89.8 \%$ de las mujeres afectadas, la edad media es de 66.8 años [5]. El antecedente de un factor estresante emocional o físico es una característica típica de este síndrome. La cirugía y la etapa perioperatoria entran como uno de estos factores [6].

Si bien las alteraciones segmentarias de la contractilidad se encuentran en los segmentos medios y apicales, se han descrito múltiples variantes de este síndrome, por lo que no podría descartase sobre todo en el segundo caso presentado. Sin embargo esta entidad generalmente aparece en el contexto de un infarto de miocardio sin obstrucción de las arterias coronarias, MINOCA por sus siglas en inglés "Myocardial infarction with non obstructive coronary arteries" [7].

En base al descarte de los posibles diagnósticos diferenciales de un cuadro de ICA en pacientes con corazón estructuralmente sano. Surge la posibilidad de estar frente a un cuadro de ICA relacionado con el uso de propofol y fentanilo.

En el primer caso el cuadro clínico de ICA y los hallazgos en el ecocardiograma concuerdan con estudios realizados que indican el efecto depresor miocardico del propofol, el mismo que en animales está relacionado con alteraciones intrínsecas de la función contráctil del miocito, antagonismo de beta receptores miocárdicos, unión a los canales de $\mathrm{Ca}$ y fosforilaciónoxidativa mitocondrial alterada [8]. El estudio realizado por Yang [9] evidenció una reducción de la contracción auricular diastólica y septal en un $21 \%$ y de la velocidad de movimiento descendente sistólico mitral anular en el segmento septal y lateral en un $26 \%$ y $29 \%$, no se evidenció reducciones significativas de la FEVI.

En el segundo caso, existe una posible relación de la bradicardia con la insuflación del neumoperitoneo, sin embargo éste generalmente no altera significativamente la frecuencia cardiaca, ya que el aumento compensatorio previsible por disminución 
del retorno venoso, gasto cardíaco e índice cardiaco es opuesto a la reacción de los barorreceptores y a la respuesta vagal secundaria a la dilatación del peritoneo y manipulación del mesenterio. El propofol puede actuar bloqueando el sistema de conducción cardiaca al actuar sobre los receptores muscarínicos colinérgicos, se reporta una incidencia de bradicardia alrededor de 4.8\% [10]. Diferentes concentraciones plasmáticas de propofol provocan efectos divergentes, bajas concentraciones alteran mínimamente el sistema de conducción, pero concentraciones altas pueden bloquear el nodo aurículo ventricular y originar arritmias supraventriculares por reentrada. Pero se requieren más estudios para atribuir un efecto directo a favor de la generación de arritmias [11]. El neumoperitoneo no ocasionó mayores efectos en el transoperatorio como grandes ascensos de $\mathrm{CO} 2$ espirado, disminución del voltaje del complejo QRS, hipotensión, incremento de presiones inspiratorias por disminución de la compliance, que obliguen a disminuir las presiones del neumoperitoneo; por ello creemos que el efecto directo del propofol sobre las células miocárdicas fueron las que influyeron en la producción de ICA, con alteraciones en la motilidad regional, aquinesia regional. La caída de la FEVI $30-33 \%$ pudo deberse al efecto del propofol aunque se ha visto en casos estudiados con ecografía reducciones del índice cardiaco pero que no suelen alterar la fracción de eyección [9].

Los casos presentaron un cuadro clínico con algunas características semejantes a un síndrome relacionado con la infusión de propofol o PRIS por sus siglas en inglés (Propofol Related Infusion Syndrome). Una rara complicación que pese a diferentes definiciones según la literatura la mayoría coincide en: colapso cardiovascular, arritmias cardiacas, y acidosis metabólica; algunas otras características comunes pero no consistentes como rabdomiolisis, hiperlipemia e hipertrigliceridemia [12].

La incidencia según las series estudiadas oscila entre 1.1 y $4.1 \%$ según Roberts [13] y Díaz [14] respectivamente, sin embargo es muy difícil estimarla pues los criterios de inclusión son aún muy divergentes, así la FDA (Food and DrugAdministration, por sus siglas en inglés) en un análisis sistemático de 1139 casos sospechosos de PRIS, verificó que aproximadamente el $30 \%$ podrían coincidir con casos verdaderos, pero con los criterios actuales es muy difícil estimar la incidencia pues ante síntomas tempranos de PRIS la infusión debe ser suspendida, pero si estos son considerados, la incidencia seria aproximadamente un $30 \%$, demostrado en el estudio de Fong [15]. La mortalidad siempre es superior al $33 \%$, y cuando se asocia a DCA incrementa a $64 \%$, según el estudio de Michael Mayette [16]. Otros estudios reportan complicaciones graves relacionadas a una inyección única de propofol con hiperkalemia, disfunción y parada cardiaca. Merz [17] y Liolios [18] afirman que el PRIS puede presentarse en infusiones de corta duración.

La etiología del PRIS aún es desconocida, no se ha demostrado los mecanismos fisiopatológicos responsables, pero pueden estar relacionados con la inhibición de la producción intracelular de energía por la mitocondria, debido a inhibición de transporte de ácidos grasos de cadena larga dentro de las células durante los estados deficientes de nutrición. Los efectos inhibitorios en la cadena respiratoria mitocondrial intracelular; provocados por una combinación de estrés metabólico y alta demanda de energía con gran utilización de oxígeno, debido a que el propofol incrementa la malonylcarnitina deteriorando la oxidación mitocondrial de los ácidos grasos libres de cadena larga, tanto en el musculo esquelético como el miocardio; los pacientes críticos dependen de estos ácidos grasos para la producción de energía y la generación de estos es dependiente de catecolaminas [8].

La asociación de propofol y el fentanilo como causa de ICA severa ha sido descrito anteriormente por Chow [19] y Renilla [2]. Tanto el propofol como el fentanilo pueden causar depresión cardiovascular y disminución de la presión arterial. El propofol dilata las arterias induciendo la síntesis de óxido nítrico, bloquea los canales de calcio, y activa la proteína quinasa $\mathrm{C}$. Lo cual en conjunto, lleva a una disminución de la precarga y a una disminución del gasto cardíaco. Este efecto ocurre con más frecuencia cuando se usa en combinación con fentanilo, en pacientes con o sin enfermedad cardíaca previa [20]. Esta combinación podría desencadenar un cuadro de ICA en pacientes con susceptibilidad individual. Cuando estos agentes se usan en combinación, se deben tomar precauciones adicionales en todos los pacientes incluyendo aquellos con corazón estructuralmente sano, como ocurrió en estos casos presentados.

A pesar de que se ha descrito que el midazolam intravenoso puede disminuir transitoriamente la pre- 
sión arterial se ha demostrado que no tiene efecto o un efecto muy leve en el gasto cardiaco y resistencias vasculares [21-24].

El atracurio debido a la liberación de histamina también produce un leve descenso de la tensión arterial con bradicardia seguida de taquicardia transitoria siendo una complicación poco frecuente dependiente de la dosis y velocidad de administración (mayor o igual a 3 veces la dosis efectiva), este efecto es antagonizado con inotrópicos o vasopresores como efedrina, fenilefrina y/o atropina [25-26]. Por ello el propofol de ser posible no debería usarse por más de tres días. El estudio realizado por Oztürk y colaboradores [27], evaluó 40 pacientes en 2 grupos grupo $\mathrm{T}$ propofol con remifentanil y grupo $\mathrm{C}$ remifentanil con sevofluorano, monitorizo: gases sanguíneos, triglicéridos, creatinquinasa, electrolitos en particular el potasio, niveles séricos de lactato, pruebas de función hepática, nitrógeno ureico sanguíneo, creatinina, CK-MB, mioglobina, troponina, carnitina total y triglicéridos cada 2 horas en el intraoperatorio y cada 6 horas en el posoperatorio y únicamente demostró un discreto aumento de los triglicéridos que descendieron a las 6 horas y contrariamente las troponinas se elevaron discretamente en el grupo $C$ por lo que el propofol sigue siendo un fármaco seguro para esta técnica. Sin embargo varios autores recomiendan usar sedaciones en la unidad de cuidados intensivos con combinación de medicamentos y no solo propofol [14].

La utilidad de monitorizar creatinquinasa ha sido cuestionada y la hipertrigliceridemia puede ocurrir en pacientes sanos luego de varias horas. A pesar de ello, el Colegio Americano de Cuidado de Medicina Crítica recomienda medir el nivel de triglicéridos después de 48 horas de iniciada la infusión, el comité europeo monitorizar en búsqueda de acidosis metabólica, rabdomiolisis, hiperkaliemia y disfunción cardiaca [16, 28].

El tratamiento de ICA asociada al uso de agentes anestésicos, incluye el rápido reconocimiento de los signos tempranos y una vez instaurado, medidas de soporte: uso de inotrópicos y agentes vasoactivos, control agresivo de los electrolitos, hemodiálisis o hemofiltración para remover el agente causal, en las formas severas con presencia de arritmias hasta la asistolia se debe considerar el soporte circulatorio mecánico con circulación extracorpórea como medida extrema al haber fracasado otras medidas [1].

\section{CONCLUSIONES}

En los casos presentados de pacientes quirúrgicos con un corazón estructuralmente sano se suscita un cuadro de insuficiencia cardiaca aguda muy probablemente debido al uso de fármacos anestésicos como el propofol y el fentanilo, una vez descartadas alteraciones estructurales.

\section{ASPECTOS BIOÉTICOS}

El presente trabajo se realizó bajo el consentimiento informado de la paciente, con total confidencialidad de datos personales.

\section{INFORMACIÓN DE LOS AUTORES}

- Cevallos Sacoto Francisco Antonio. Especialista en anestesiología y reanimación. Hospital Vicente Corral Moscoso.

ORCID: https://orcid.org/0000-0001-7416-1956

- Puga Bravo Cristina Evelyn. Especialista en anestesiología y reanimación. Hospital Isidro Ayora de Loja.

ORCID: https://orcid.org/0000-0001-7301-3804

- Serrano Béjar Leonardo Alfredo. Médico. Postgradista en Anestesiología. Universidad de Cuenca. ORCID: https://orcid.org/0000-0002-7347-0754

- Tirapé Castro Hugo Arturo. Médico. Hospital Vicente Corral Moscoso.

ORCID: https://orcid.org/0000-0002-5962-3830

\section{CONTRIBUCIÓN DE LOS AUTORES}

FACS: revisión bibliográfica, discusión, introducción, conclusiones, confección y modificaciones. CEPB: recolección y resumen de casos, revisión bibliográfica, conclusiones, traducción inglés, ortografía, revisión de filología. LASB: revisión bibliográfica. HATC: revisión bibliográfica

\section{CONFLICTO DE INTERESES}

Los autores declaran no tener conflicto de intereses en la presente investigación.

\section{FUENTES DE FINANCIAMIENTO}

Ninguna 


\section{REFERENCIAS BIBLIOGRÁFICAS}

1. Ponikowski $\mathrm{P}$, Voors $\mathrm{A}$, Anker $\mathrm{S}$, Bueno $\mathrm{H}$, Cleland J, Coats A, et al. Guía ESC 2016 sobre el diagnóstico y tratamiento de la insuficiencia. RevEspCardiol. 2016; 69(12): 1167.e1-e85.

2. Renilla González A, Lozano MartínezLuengas I, Secades González S, Álvarez Pichel I, Álvarez Martínez P, Santamarta LE, et al. Cardiogenic shock following administration of propofol and fentanyl in a healthy woman: a case report. Journal of Medical Case Reports. 2011; 5: 382. Disponible en: https://www.ncbi. nlm.nih.gov/pubmed/21846377

3. PollackA, Kontorovich A, Fuster V, Dec G. Viral myocarditis-diagnosis, treatment options, and current controversies. NatRevCardiol. 2015; 12(11): 670-80. Disponible en: https://www. ncbi.nlm.nih.gov/pubmed/26194549

4. Dinic V, Markovic D, Savic N, Kutlesic M, Jankovic R. Peripartum Cardiomyopathy in Intensive Care Unit: An Update. Front Med (Lausanne). 2015; 2: 2. Disponible en: https://www.ncbi.nlm.nih.gov/pmc/articles/ PMC4655438/

5. Templin C, Ghadri J, Diekmann J, Napp L, Bataiosu D, Jaguszewski $M$, et al. Clinical features and outcomes of Takotsubo (stress) cardiomyopathy. N Engl J Med. 2015; 373:929-38. Disponible en: https://www.ncbi. nlm.nih.gov/pubmed/26332547

6. Kato K, Lyon A, Ghadri J, Templin C. Takotsubo syndrome: aetiology, presentation and treatment. Heart. 2017; 103:1461-1469. Disponible en: https://www.ncbi.nlm.nih.gov/ pubmed/28839096

7. Poku N, Noble S. Myocardial infarction with non obstructive coronary arteries (MINOCA): a whole new ball game. ExpertRevCardiovascTher. 2017; 15(1): 7-14. Disponible en: https://www.ncbi.nlm.nih.gov/ pubmed/27892770

8. Vollmer J, Haen S, Wolburg H, Lehmann R, Steiner J, Reddersen S, et al. Propofol Related Infusion Syndrome: Ultrastructural Evidence for a Mitochondrial Disorder. Crit Care Med.
2018; 461: e91-e94. Disponibleen: https:// www.ncbi.nlm.nih.gov/pubmed/29252954

9. Yang H, Song B, Kim J, Kim S, Kim T. Impact of propofol anesthesia induction on cardiac function in low-risk patients as measured by intraoperative Doppler tissue imaging. J Am SocEchocardiogr. 2013; 26(7): 727-35. Disponible en: https://www.ncbi.nlm.nih.gov/ pubmed/23622885

10. Schroeppel T, Clement L, Barnard D, Guerrero W, ferguson M, Sharpe J, et al. Propofol Infusion Syndrome: Efficacy of a Prospective Screening Protocol. Am Surg. 2018; 84(8): 1333-8.

11. Liu Q, Kong A, Chen R, Qian C, Liu S, Sun $B$, et al. Propofol and arrhythmias: two sides of the coin. Acta Pharmacol Sin. 2011; 32(6): 817-23. Disponible en: https://www.ncbi.nlm. nih.gov/pubmed/21642950

12. Agrawal N, Rao S, Nair R. A death associated with possible propofol infusion syndrome. Indian J Surg. 2013; 75(Suppl 1): 407-8. Disponible en: https://www.ncbi.nlm.nih.gov/ pmc/articles/PMC3693369/

13. Roberts R, Barletta J, Fong J, Schumaker G, Kuper P, Papadopoulos S, et al. Incidence of propofol-related infusion syndrome in critically ill adults: a prospective, multicenter study. CritCare. 2009; 13(5): R169 (doi:10.1186/ cc8145). Disponible en: https://www.ncbi.nlm. nih.gov/pubmed/19874582

14. Díaz J, Prabhakar A, Urman R, Kaye A. Propofol infusion syndrome: a retrospective analysis at a level 1 trauma center. Crit Care Res Pract. 2014; 2014:346968. doi: 10.1155/2014/34696. Disponibleen: https:// www.ncbi.nlm.nih.gov/pubmed/25580289

15. Fong J, Sylvia L, Ruthazer R, Schumaker G, Kcomt M, Devlin J. Predictors of mortality in patients with suspected propofol infusion syndrome. CritCareMed. 2008; 36(8): 2281-7. Disponible en: https://www.ncbi.nlm.nih.gov/ pubmed/18664783

16. Mayette M, Gonda J, Hsu J, Mihm F. Propofol infusion syndrome resuscitation with extracorporeal life support: a case report and 
review of the literature. Ann IntensiveCare. 2013; 3(1): 32. Disponible en: https://www. ncbi.nlm.nih.gov/pubmed/24059786

17. Merz T, regli B, Rothen H, Felleiter P. Propofol infusion syndrome a fatal case at a low infusion rate. AnesthAnalg. 2006; 103(4): 1050. Disponible en: https://www.ncbi.nlm. nih.gov/pubmed/17000843

18. Liolios A, Guérit J, Scholtes J, Raftopoulos C, Hantson P. Propofol infusion syndrome associated with short-term large-dose infusion during surgical anesthesia in an adult. AnesthAnalg. 2005; 100: 1804-6. Disponible en: https://www.ncbi.nlm.nih.gov/ pubmed/15920217

19. Chow S, Houseman D, Phug T, French W. Transient acute decompensated heart failure following propofol and fentanyl administration in a healthy 19 -year-old patient. Congest Heart Fail. 2010; 16: 80-1.Disponible en: https:// www.ncbi.nlm.nih.gov/pubmed/20412475

20. Larsen J, Torp P, Norrid K, Sloth E. Propofol reduces tissue-Doppler markers of left ventricle function: a transthoracic echocardiographic study. Br J Anaesth. 2007; 98: 183-8.

21. Forster A, Gardaz JP, Suter PM, Gemperle M (1980) I-V midazolam as an induction agent for anesthesia: a study in volunteers. $\mathrm{Br} \mathrm{J}$ Anaesth 52:907-911.

22. Lebowitz PW, Cote ME, Daniels $A L$, et al. (1982) Comparative cardiovascular effects of midazolam and thiopental in healthy patients. AnesthAnalg 61:771-775.

23. Al-Khudhairi D, Whitwam JG, Chakrabarti MK, Askitopoulou H, Grundy EM, Powrie S (1982) Haemodynamic effects of midazolam and thiopentone during induction of anaesthesia for coronary artery surgery. Br J Anaesth 54:831-835.

24. Marty J, Nitenberh A, Blanchet F, Zouioueche S, Desmonts JM (1986) Effects of midazolam on the coronary circulation in patients with coronary artery disease. Anesthesiology 64:206-210.

25. Correa Claudia MN, Sudo GZ, Sudo RT. Efeitoshemodinâmicos do atracúrio e do cisatracúrio e o uso de difenidramina e cimetidina. Rev. Bras. Anestesiol. [Internet]. 2010 Feb [Citado: 2019Apr 24] ; 60( 1 ): 52-63. Disponible en: http://www.scielo.br/ scielo.php?script $=$ sci_arttext\&pid=S003470942010000100006\&lng=en. http://dx.doi. org/10.1590/S0034-70942010000100006.

26. Lien CA, Belmont MR, Abalos A et al. The cardiovascular effects and histaminereleasing properties of 51W89 in patients receiving nitrous oxide/opioid barbiturate anesthesia. Anesthesiology 1995;82:11311138

27. Oztürk I, Serin S, Gürses E. Biochemical markers in total intravenous anesthesia and propofol infusion syndrome: a preliminary study. EurRevMedPharmacolSci. 2018; 17(24): 3385-90. Disponible en: https://www. ncbi.nlm.nih.gov/pubmed/24379072

28. Imam T. Propofol-related infusion syndrome: role of propofol in medical complications of sedated critical care patients. Perm J. 2013; 17(2): 85-7. Disponible en: https://www.ncbi. nlm.nih.gov/pmc/articles/PMC3662290/ 